\title{
In vitro studies on the effect of 8-(4-chlorophenylthio) cAMP on selected biochemical parameters in Sertoli cells isolated from testis of transgenic mice
}

\author{
M. Bhaskar \\ Department of Zoology, S.V. University, Tirupati- 517 501, A.P. India
}

\begin{abstract}
The central objective of present study was to know the effect of 8-(4-chlorophenylthio) cyclic 3' 5 adenosine monophosphate (cpt-cAMP: $5000 \mathrm{ng} / \mathrm{ml}$ ) on selected biochemical parameters in Sertoli cells isolated from testis of mice over-expressing bovine growth hormone. The results indicated that cpt-cAMP treated Sertoli cells isolated from mice over-expressing $b G H$ showed a significant increase $(p<0.001)$ in the levels of total proteins (33.78\%), DNA (167.81\%), RNA (152.38\%) and lactic acid (49.56\%) levels as compared to treated normal Sertoli cells over a period of five days. However, surprisingly, cpt-cAMP-induced effects in Sertoli cells isolated from testis of transgenic and normal mice were significantly lower as compared to their respective controls. From the results, it can be concluded that cpt-cAMP-induced changes in selected biochemical variables might indicate reversal effects against GH-regulation of protein synthesis and lactate production. These results provide basic information related to the response of GH against excess levels of second messengers in the regulation of metabolic fate of Sertoli cells.
\end{abstract}

KEYWORDS:Cpt-cAMP, DNA, Lactic Acid, Pepck-bGH Transgenic Mice, RNA, Sertoli Cells, Total Proteins

\section{INTRODUCTION}

Growth hormone plays an important role in hormone replacement therapy (HRT) to improve fertility among men and also to sustain animal husbandry. GH is gaining popularity day-by-day as a potential therapeutic candidate to ameliorate spermatogenesis among men at reproductive age with suppressed sperm production and/or sperm maturation events. Though, the exact mechanism of action of GH-induced ameliorated spermatogenic effects are poorly understood, many studies claimed that GH interferes and modulates endocrine factors of hypothalamo-pituitary-testicular (HPT) axis. Thus, a cross-talk between gonadotropins such as follicle stimulating hormone (FSH), leutinizing hromone (LH), testosterone and GH might be responsible for accelerated spermatogenic effects [1-4]. Furthermore, some preliminary data in vitro suggested that GH positively modulates Sertoli cell DNA synthesis, targets machinery of transcription and translation to trigger protein synthesis and also provides adequate levels of energy through lactate for sertoli cells thereby promote spermatogenesis [5, 6]. Thus, it is apparent that GH has ability to modulate the metabolic fate of sertoli cells and influence spermatogenesis.

In addition to hormones, many secondary signaling molecules also play pivotal roles in the regulation of male reproduction . Cyclic 3 $\square, 5 \square$ adenosine monophosphate [cAMP) is one of the classical second messengers with regulatory effects on sperm production and maturation. Earlier, it has been indicated that cAMP through its response element target regulation of expression of genes required for spermiogenesis and influence sperm maturation [7,8]. Apart from its direct effects, cAMP acts as signaling molecule for FSH to affect spermatogenesis [8]. Though, chemical nature, mode of action and target sites are well documented for cAMP, its effects on basic biochemical machinery in Sertoli cells are poorly understood. It is also intriguing both GH and cAMP production at least in part induced by gonadotropin hormone releasing hormone secreted from pituitary gland [9]. Thus, studies which address the effects of cAMP and GH on basic biochemical events that occur in Sertoli cells provide valuable information regarding mechanistic and interactive effects of GH. Therefore, the aim of the present study was to investigate the effect of cAMP analog, on biochemical events in Sertoli cells isolated from testis of mice (PEPCK-bGH) over-expressing bovine growth hormone. This animal model was selected because; it is widely used mice to understand the physiological effects of GH and moreover, it serves as good experimental model to study reproductive functions which mimic a model with supra-normal physiological levels of GH [10]. 


\section{MATERIALS AND METHODS}

\subsection{Animals}

For the present study, male transgenic animals derived from a single male founder produced by microinjection of the phosphoenol pyruvate carboxykinase (PEPCK) promoter region (300-bp) bGH, hybrid gene into the male pro-nucleus of single cell embryos were selected and this breeding system resulted in a production of approximately equal number of hemizygous transgenic and normal (Non-Transgenic) offspring. The nontransgenic mice were referred to as controls. All the animals were fed ad libitum a standard laboratory rodent diet (Teklad 6\% rat/mouse diet, 002'; Herlan Sprague Dawley, Madison W.I; 24\% protein, 6\% fat) and tap water. Litters were weaned between 20 and 22 days of age (day of birth $=$ day ' 0 '). The identification and selection of these animals were based on earlier reports [11].

\subsection{Experimental design}

Prior to the isolation and culture studies, testis were immediately removed and weighed to its nearest milligram. After, weighing, the isolation and culture of Sertoli cells from testis of transgenic and non-transgenic mice was based on Welsh and Wiebe [12). Briefly, approximately 200 clusters per $2 \mathrm{ml}$ of incubation media containing antibiotics (200 U penicillin/ml, $200 \mathrm{ng}$ streptomycin/ml, $0.5 \mathrm{ng}$ amphotericin B/ml and $100 \mu \mathrm{g}$ gentamycin/ml) and 8-(4-chlorophenylthio) cAMP $(5000 \mathrm{ng} / \mathrm{ml})$ were plated in $35 \mathrm{~mm}$ pre-marked dishes and incubated in an atmosphere of 5\% CO2 $+95 \%$ air and at $34 \pm 10 \mathrm{C}$ temperature. All the reagents used in the present study were purchased from Sigma Co. Ltd., USA. The day of plating was considered as day 0 of culture and the dishes were not disturbed until day 2 of culture. After completion of two days, the dishes were shaken gently and fresh incubation medium was replaced. The percent spreading, maintenance capacity and culture characteristics was determined on day 4 of culture was performed according to Bhaskar [6]. Total proteins, lactic acid, DNA and RNA from normal and transgenic Sertoli Cells were determined by methods described previously [13-15]. All the biochemical parameters were performed in isolated Sertoli cells from normal and transgenic mice treated with or without cAMP analog and moreover, the results were compared to our earlier findings [6], in order to assess the interactive effect of cAMP analog and GH in the regulation of metabolic fate of Sertoli cells isolated from testis of both transgenic and normal mice.

\subsection{Statistical Analysis}

Differences between groups were analyzed for statistical significance by using student's t-test on raw data. $p<0.001$ was considered as significant.

\subsection{Toxicity}

\section{RESULTS}

All the animals used in the present study showed no clinical signs of toxicity.

\subsection{Effect of cAMP analog on selected biochemical variables in Sertoli cells isolated from control and transgenic mice}

Figure 1 represents the changes in the levels of DNA, RNA, total proteins and lactic acid of Sertoli cells isolated from testes of transgenic and normal mice. In the present study, significant $(\mathrm{p}<0.001)$ increase in the levels of total proteins, lactic acid, DNA and RNA were observed in transgenic Sertoli cells treated with cAMP as compared to normal Sertoli cells treated with cAMP analog. The percent increase in total proteins (Fig. 1A), RNA (Fig. 1B), DNA (Fig. 1C), and lactic acid (Fig. 1D) in transgenic Sertoli cells as compared to normal Sertoli cells were $33.78,167.81,152.38$ and 49.56 , respectively.

\subsection{Changes in selected biochemical variables in Sertoli cells isolated from testis of control and transgenic mice during different experimental conditions}

Figure 2 depicts the changes in selected biochemical variables in the Sertoli cells isolated from testis of mice during different experimental conditions. cAMP treated Sertoli cells isolated from testis of control and transgenic mice showed a significant decrease as compared to their respective controls. The percent decrease in the levels of DNA (49.39), RNA (45.05), total proteins (50.11) and lactic acid levels (47.61) in cAMP treated normal Sertoli cells as compared to untreated Sertoli cells. Similar trend was observed in the levels of DNA (51.41), RNA (-22.31), total proteins (-67.09) and lactic acid (-63.41) levels in cAMP treated transgenic Sertoli cells as compared to untreated transgenic Sertoli cells. 


\section{DISCUSSION}

The findings of the present study clearly demonstrated that exposure of transgenic Sertoli cells to cAMP analog showed a significant increase (Fig. 1) in the levels of DNA, RNA, lactic acid and total proteins as compared to cpt-cAMP treated normal Sertoli cells. However, cpt-cAMP-induced changes in selected biochemical variables of transgenic and normal Sertoli cells were significantly lower as compared to their respective controls (Fig. 2). The decrease in the levels of DNA and RNA in cpt-cAMP exposed transgenic Sertoli cells might indicate decreased DNA synthesis and reduced transcriptional activity, respectively. Nucleic acids such as DNA and RNA are believed to be important vital components of the cell which reflect the total protein levels during spermatogenesis. The decrease in the total proteins and lactate levels in transgenic and normal Sertoli cells exposed to cpt-cAMP might indicate the regulatory effects of cpt-cAMP at the level of translation and energy metabolism. The other biochemical variable, lactate is well known for its role in the regulation of spermatogenesis. It regulates spermatogenesis by serving as a transcription survival factor and participates in mitochondrial oxidative phosphorylation for the production of ATP which is required for developing germ cells [16].

It is well known that mechanisms of intrinsic and extrinsic chemical moieties require a cross-talk between endocrine and non-endocrine factors such as cAMP to amplify and transmit signals to influence molecular and physiological events for a variety of cellular functions to occur in a timely precise manner. Thus, cAMP, is an important second messenger that carries signals for a range of chemical moieties. It exerts its effects by activating the cAMP-dependent protein kinaseA (PKA), which transduces the signal through phosphorylation of different target proteins. In the present study, cAMP exposure showed a significant decrease in the total protein levels in Sertoli cells isolated from testis of transgenic and normal mice, indicating cAMPinduced regulatory effects on transcription or translation and/or both [17]. The other biochemical variable lactate plays a vital role in energy homeostasis by acting as ATP producing moieties during mitochondrial oxidative phosphorylation. In the present study, cAMP exposure significantly reduced the lacate levels in Sertoli cells isolated from transgenic and normal mice as compared to their respective controls. This reflects cAMP-induced effects on energy utilization processes. One of the plausible reasons for this might be due to the fact that cAMP activated protein kinase subuints have ability to regulate lactate dehydrogenase (LDH), an enzyme which involves in the concomitant oxidation/reduction of NADH to NAD thereby inter-conversion of pyruvate to lactate. [18]. Though, LDH levels did not performed in the present study, the decreased levels of lactate levels in both transgenic and normal Sertoli cells exposed to cAMP might be attributed to its regulatory effects at the level of LDH.

Piecing these results, it can be concluded that, cAMP exposure showed inhibitory effects on DNA, RNA, protein and lactate levels in Sertoli cells isolated from testis of normal and transgenic mice and moreover, it can also be suggested that cAMP showed reversal effects on GH-induced increased levels of selected biochemical variables in transgenic Sertoli cells in vitro.

\section{CONCLUSION}

The findings of the present study throw a light on the interactions between GH and cAMP in the regulation of DNA synthesis, transcription and translation activities and also energy metabolism that occur in Sertoli cells in vitro. However, future experiments exploiting spermatogenic regulatory factors which mediate cAMP (eg: FSH) are necessary to get a clear cut picture for effective use of GH as a potential therapeutic candidate to sustain animal husbandry and also in HRTs to ameliorate fertility among men with suppressed spermatogenesis.

\section{ACKNOWLEDGEMENTS}

This study was supported by Rockefeller Foundation, New York, USA (Ref: 93022). The author is thankful to Dr. J.N. Rao, Dr. V. Chandrasekhar and Dr. Michael Collard for sharing their experience in culture of Sertoli cells and completing the work. The author also acknowledges Dr. Andrej Bartke, for his mentoring and providing laboratory facilities in the Department of Physiology, School of Medicine, Southern Illinois University, Carbondale, IL-62901, USA. 
Figure 1: $\quad$ Changes in the levels of total proteins (A), RNA (B), DNA (C) and lactic acid (D) in cpt-cAMP treated transgenic and normal Sertoli cells

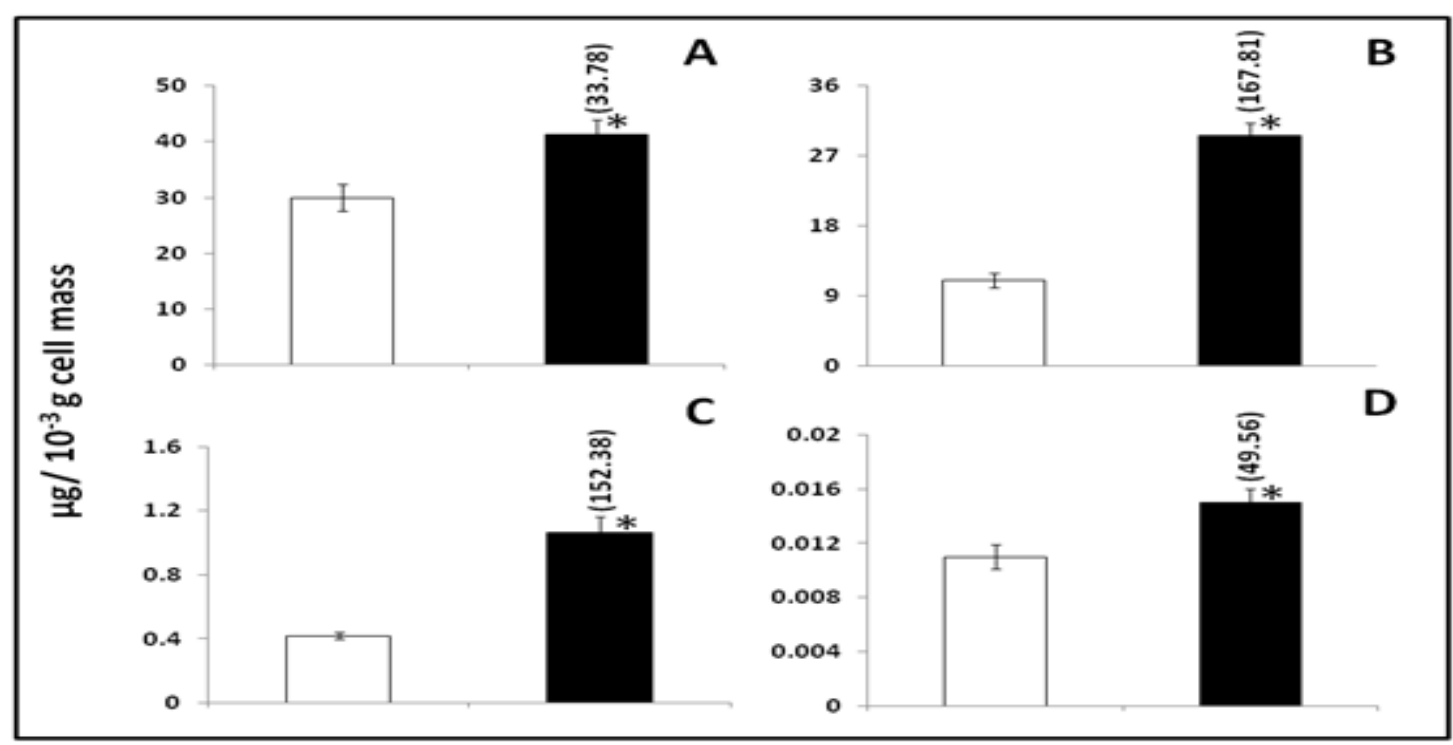

Bars are mean \pm S.D. Values in the parentheses are percent change values.For evaluation of 'p' and \% change, for cpt-cAMP treated transgenic Sertoli cells (solid bars), cpt-cAMP treated normal Sertoli cells (open bars) served as controls. *significant at $\mathrm{p}<0.001$.

Figure 2: Changes in the levels of selected biochemical variables in cpt-cAMP treated transgenic and normal Sertoli cells against normal and transgenic Sertoli cells

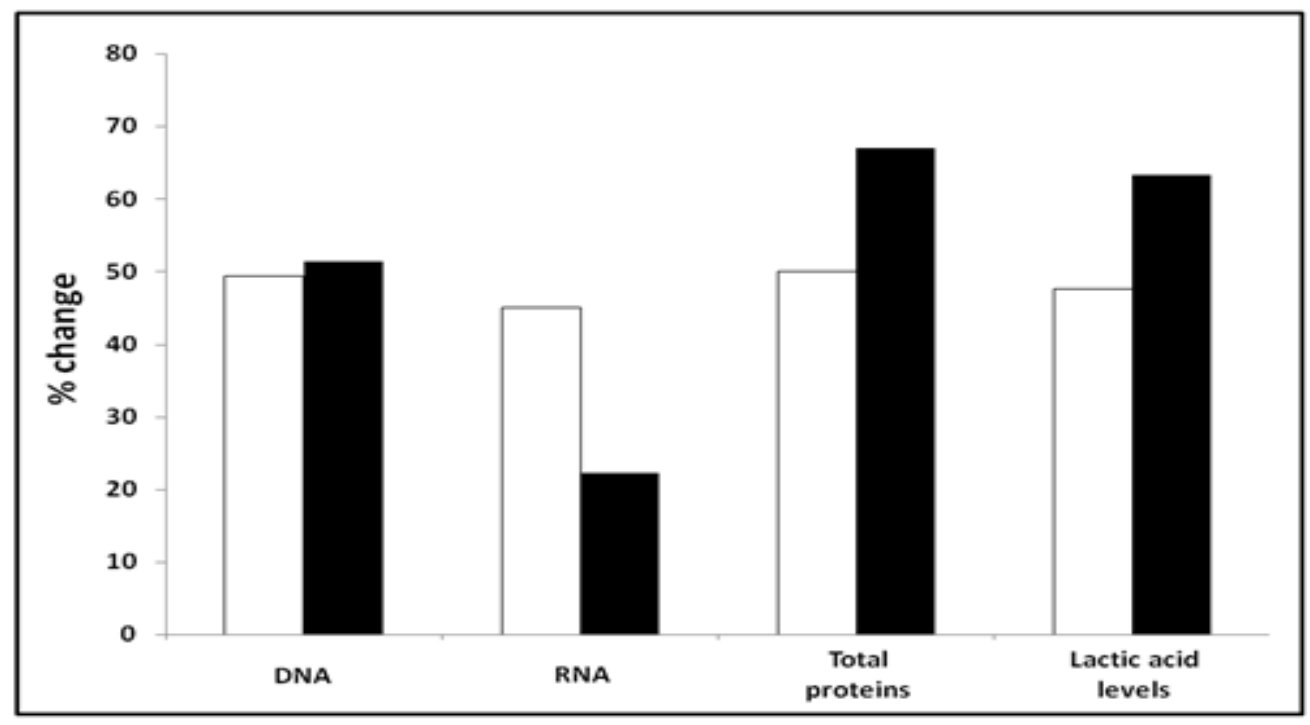

Bars indicate percent decrease values. For evaluation of $\%$ change, for cpt-cAMP treated transgenic Sertoli cells (solid bars), untreated transgenic Sertoli cells served as controls and for cpt-cAMP treated normal Sertoli cells (open bars), untreated Sertoli cells served as controls. 


\section{REFERENCES}

[1.] N. Magon, S. Singh, A. Saxena and R. Sahay, Growth hormone in male infertility, Indian Journal of Endocrinology and Metabolism $15,2011,248-249$.

[2.] A. Bartke, V. Chandrashekar and R.W. Steger, Effects of growth hormone on neuroendocrine function, Acta Neurobiologiae Experimentalis 56, 1996, 833-842.

[3.] D. Madhukar, and S. Rajender, Hormonal treatment of male infertility: Promises and pitfalls, Journal of Andrology 30, 2009, 95112 .

[4.] S. Kalra, B. Kalra and A. Sharma, Growth hormone improves semen volume, sperm count and motility in men with idiopathic normogonadotropic infertility, Endocrinology Abstracts 16, 2008, 613.

[5.] A. Bartke, E.M. Naar, L. Johnson, M.R. May, M. Cecim, J.S. Yun, and T.E. Wagner, Effects of expression of human or bovine growth hormone genes on sperm production and male reproductive performance in four lines of transgenic mice, Journal of Reproduction and Fertility 95, 1992, 109-118.

[6.] M. Bhaskar, In vitro studies on changes in selected biochemical parameters and morphology of Sertoli cells in mice overexpressing bovine growth hormone, IOSR Journal of Pharmacy 3, 2013, 43-48.

[7.] P. Sassoni- Cori, Transcription factors responsive for cAMP, Annual Reviews of Cellular and Developmental Biology 11, 1995, 335-377.

[8.] R. Behr, G.F. Weinbauer, cAMP response element modulator (CREM): an essential factor for spermatogenesis in primates? International Journal of Andrology 24, 2001, 126-135.

[9.] J. E. Horváth, K .Groot, A.V. Schally, Growth hormone-releasing hormone stimulates cAMP release in superfused rat pituitary cells, Proceedings of National Academy of Sciences U S A 92, 1995, 1856-1860.

[10.] M.G. Castro, Transgenic models in endocrinology, (USA: Kluwer Academic Publishers, 2001).

[11.] A. Bartke, E.M. Naar, L. Johnson, M.R. May, M. Cecim, J.S. Yun, and T.E. Wagner, Effects of expression of human or bovine growth hormone genes on sperm production and male reproductive performance in four lines of transgenic mice, Journal of Reproduction and Fertility 95, 1992, 109-118.

[12.] M.J. Welsh, J.P. Wiebe, Rat Sertoli cells: A rapid method for obtaining viable cells, Endocrinology 96, 1975, 618-624.

[13.] K.W. Giles, and A. Myers, An Improved Diphenylamine Method for the Estimation of Deoxyribonucleic Acid, Nature 206, 1965, 93.

[14.] M. Bradford, A Rapid and Sensitive Method for the Quantitation of Microgram Quantities of Protein Utilizing the Principle of Protein-Dye Binding, Analytical Biochemistry 72, 1976, 248-254.

[15.] H.J. Hohorst, L(+)-Lactate: Determination with lactic dehydrogenase and DPN. In H.U. Bergmeyer (Ed.), Methods of Enzymatic Analysis, (Weinheim: Verlag Chemie, 1965) 266-270.

[16.] M.F. Reira, S.B. Meroni, G.E. Gomez, H.F. Schteingart, E.H. Pellizzari and S.B. Cigorraga, Regulation of lactate production by FSH, IL1 beta and TNF alpha in rat Sertoli cells, General and Comparative Endocrinology 122, 2001, 88-97.

[17.] P.B. Daniel, W.H. Walker, J.F. Habener, Cyclic AMP signaling and gene regulation, Annual Reviews of Nutrition, 18, 1998, 353383.

[18.] R.A. Jungmann, A.I. Constantinou, S.P. Squinto, J. Kwast-Welfeld, J.S. Schweppe, Regulation of lactate dehydrogenase gene expression by cAMP-dependent protein kinase subunits, Annals of the New York Academy of Sciences 478, 1986, 147-161. 